\title{
Scalp lacerations demand careful attention before interhospital transfer of head injured patients
}

\author{
M O Fitzpatrick, K Seex
}

\begin{abstract}
Blood loss from scalp lacerations may be considerable. Two cases are described to illustrate the hazards of transferring patients with head injuries without adequate attention to scalp wounds. In such cases referring clinicians must be satisfied that haemostasis is secure. Failure to do this may place the patient at increased risk as a result of the need for additional resuscitation, and therefore delay the definitive management.

( $\mathcal{A}$ Accid Emerg Med 1996;13:207-208)
\end{abstract}

Key terms: scalp lacerations; head injury; interhospital transfer; haemorrhagic hypotension

Scalp lacerations are a common reason for attendance at accident and emergency (A\&E) departments. In most cases they are successfully managed by simple suture or stapling under local anaesthetic infiltration. The potential problem of significant blood loss appears to be obvious but is easily overlooked, since in everyday practice only a minority of scalp lacerations require hospital admission for resuscitation and blood transfusion.

Severe trauma often demands multidisciplinary management and, especially in the head injured patient, interhospital transfer. If scalp lacerations are overlooked or not managed correctly, continued blood loss can result in hypovolaemic shock. These secondary insults can lead to deterioration in the patients condition during transfer. The definitive management is then delayed since further investigations and resuscitation are necessary. This delay can result in an increase in morbidity and poor outcome.

We present two cases which were transferred to a regional neurosurgical centre after initial management at $A \& E$ level. In these patients, scalp lacerations had been noted by the attending doctor but had not been dealt with in an effective manner. The resulting problems affected the patients' management and increase the risks of a poor outcome. They were also preventable.

Neurosurgery, Institute of

Neurological Sciences, Southern General Hospital, Govan Road, Glasgow, Scotland M O Fitzpatrick K Seex

Correspondence to: Mr M O Fitzpatrick, Wellcome Surgical Institute, Garscube Estate, Glasgow G61 1Q $\mathrm{H}$ admission to the $A \& E$ department he was able to localise pain. He was haemodynamically stable and there were no other systemic injuries. A plain skull $x$ ray confirmed multiple skull fractures. Haemoglobin was within normal limits. There were no facilities on site for computerised tomography (CT). On the basis of his clinical state, transfer to the neurosurgical unit was arranged. In the ambulance he required intravenous fluid when he became hypotensive.

On arrival his blood pressure was low, with a systolic pressure of $65 \mathrm{~mm} \mathrm{Hg}$ and a pulse of 156 beats $/ \mathrm{min}$. Active resuscitation was carried out with colloid and crystalloid solutions and blood was cross matched. His haemoglobin was now $6.3 \mathrm{~g} / \mathrm{dl}$. The head bandage applied at the base hospital was saturated with blood. When it was removed it was clear there was substantial bleeding from the scalp lacerations which had not been sutured before transfer. This patient required extensive resuscitation with blood and clotting factors before he was even stable enough to be transferred to the CT room. During this delay his left pupil became fixed and dilated, necessitating emergency CT and immediate operation to evacuate a large left sided extradural haematoma.

From a controlled situation at the base hospital this patient's care had become an emergency because of delay in the neurosurgical management imposed by the need to deal with haemorrhage from the scalp lacerations, whose potential for causing massive blood loss had not been appreciated or dealt with in an adequate way.

Postoperatively the patient remained in intensive care for several days but eventually made a full recovery and returned home.

\section{Case 2}

A 27 year old woman was admitted to $A \& E$ following an assault with a knife. She was able to walk into the department and provide clinical details. She was haemodynamically stable and no neurological deficit was detected. There were multiple scalp lacerations. A plain skull $x$ ray showed a depressed skull fracture and intracranial air. She was referred to the neurosurgical unit with the diagnosis of a penetrating head injury. Before transfer, the scalp lacerations were cleaned and the edges closed with standard surgical staples by the casualty officer. At this stage haemostasis appeared under control.

Unfortunately, during transfer the patient had a grand mal seizure and became cyanosed. The accompanying doctor inserted an airway 
and manual ventilation of the patient continued throughout transfer. Intravenous phenytoin controlled seizure activity. During these procedures several staples in the scalp lacerations became dislodged and there was profuse bleeding from the wounds. Attempts were made to control the bleeding but haemostasis was not achieved.

On arrival at the neurosurgical unit the patient's condition had deteriorated. She was hypotensive (arterial pressure $70 / 40 \mathrm{~mm} \mathrm{Hg}$ ), tachycardic (heart rate 130 beats/min), and tachypnoeic. Rapid resuscitation was carried out and blood cross-matched. Haemoglobin level was $7 \cdot 1 \mathrm{~g} / \mathrm{dl}$. Over the next two hours she had a further seizure but her haemodynamic status stabilised. She was transfused with four units of packed cells. Subsequent surgery confirmed that the knife had penetrated the dura and there was a small subdural haematoma, which was evacuated. Postoperative care was uneventful and she was discharged on a maintenance dose of phenytoin.

\section{Discussion}

The problems associated with the inter hospital transfer of the head injured patient are well recognised ${ }^{1}$ and haemorraghic shock following scalp lacerations is described. ${ }^{2}$ It would seem obvious to any clinician with experience in emergency departments that scalp lacerations have the potential for profuse blood loss and that their correct management demands secure haemostatic control. This usually involves simple suturing with an appropriate material. Other acceptable methods include surgical staples ${ }^{3}$ or more specialised scalp clips, ${ }^{4}$ the crucial point being that good, secure haemostasis is achieved.

If haemostasis is not achieved a cascade of events can follow, with resultant deterioration in the patient's clinical state. This is especially dangerous during interhospital transfer, which is a common stage in the management of the head injured patient requiring neurosurgical assessment. The head injured patient needs prompt investigation and treatment if secondary insults to the brain are to be avoided. Hence, reducing the period between admission and definitive care should be facilitated by doing the simple things efficiently and to a high standard.

With good technique the time taken to correctly manage scalp lacerations should not in itself introduce a significant delay. It is better to delay transfer and achieve haemostasis, since the clinical deterioration of the patient during transfer could have serious consequences and itself result in significant delay.

We suggest that the potential for scalp lacerations to cause haemorraghic hypotension should be emphasised to all clinicians. Furthermore, if interhospital transfer is indicated the referring clinician must be satisfied that haemostasis is secure. Haemostatic procedures must also be able to withstand the transfer and the management of any complications which could be anticipated. Failure to follow these basic principles may place the patient at increased risk as a result of the need for additional resuscitation, and therefore delay the definitive management.

1 Gentleman D, Dearden M, Midgley S, MacLean D Guidelines for resuscitation and transfer of patients with Guidelines for resuscitation and transfer of
serious head injury. $B M \mathcal{F} 1993 ; 307: 547-52$.

2 Lemos MJ, Clark DE. Scalp lacerations resulting in haemorrhagic shock: case reports and recommended haemorrhagic shock: case reports and

3 Ritchie AJ, Rocke LG. Staples versus sutures in the closure of scalp wounds: a prospective double blind, randomised trial. Injury 1989;20:217-8.

4 Sykes LN, Cowgill F. Management of haemorrhage from severe scalp lacerations with Raney clips. Ann Emerg Med 1989;18:995-6.

\author{
Accident and \\ Emergency \\ Department, \\ Stockport Infirmary, \\ Stockport \\ M Saab \\ A Gray \\ D Hodgkinson \\ Neurosurgical \\ Department \\ Manchester Royal \\ Infirmary, Manchester \\ M Irfan \\ Correspondence to: \\ M Saab, \\ Senior Registrar in Accident \\ and Emergency, Stockport \\ nfirmary, Wellington Road \\ South, Stockport SK1 3UJ \\ United Kingdom.
}

\section{Warfarin and the apparent minor head injury}

\author{
M Saab, A Gray, D Hodgkinson, M Irfan
}

Key terms: anticoagulation; apparent minor head injury; intracranial haematoma; warfarin

It is estimated that 1.4 million patients each year attend accident and emergency (A\&E) departments in the United Kingdom with a head injury. The vast majority have a minor head injury which has been defined as a history of unconsciousness of less than 20 minutes, a Glasgow coma scale score of 13-15 on admission, and an inpatient stay not exceeding 48 hours. $^{1}$ Most of these patients are

\section{department.}

(f Accid Emerg Med 1996;13:208-209) 